\title{
Assimilation de données par méthode variationnelle utilisant l'adjoint d'un code de circulation côtière tridimensionnel
}

\author{
Jean-Luc DEVENON*, Ivan DEKEYSER ${ }^{\text {b }}$, Yann LEREDDE ${ }^{c}$, Jean-Michel LELLOUCHE \\ ${ }^{a}$ Laboratoire de sondages électromagnétiques dans l'environnement terrestre, UMR-CNRS 6017, \\ université de Toulon et du Var, BP132F, 83957 La Garde cedex, France \\ b UMS-CNRS 2196, Centre d'océanologie de Marseille, université Aix-Marseille-II, Campus de Luminy, \\ 13288 Marseille cedex 9, France \\ c UMR-CNRS 6535, Laboratoire d'océanographie et de biogéochimie, Centre d'océanologie de Marseille, \\ université d'Aix-Marseille II, Campus de Luminy, 13288 Marseille cedex 9, France
}

\begin{abstract}
Résumé - Une méthode de contrôle optimal est utilisée pour identifier les conditions aux limites ouvertes et les coefficients de viscosité turbulente d'un modèle tridimensionnel de circulation côtière aux équations primitives au moyen d'une méthode d'assimilation de données par méthode variationnelle. L'objectif de cette méthode est d'assurer l'optimisation des paramètres inconnus du modèle en minimisant une fonction de coût mesurant l'écart entre la solution calculée et des observations. La minimisation de cette fonction de coût est réalisée en utilisant l'adjoint du linéarisé tangent du modèle côtier. Une méthode est donnée, dans le cas d'un modèle aux différences finies utilisant une grille décentrée, pour passer de la formulation continue du problème de contrôle optimal et du modèle adjoint à une formulation discrétisée. L'efficacité de la méthode est vérifiée à partir du cas de l'ajustement des conditions aux limites ouvertes pour une circulation associée à une seiche de Kelvin dans un bassin semi-fermé. Elle est ensuite testée vis à vis de l'optimisation du champ de coefficients de viscosité turbulente dans le cas de déstructuration soudaine d'une stratification préalablement existante, comme dans le cas d'une rupture d'une halocline marquée provoquée par un coup de vent. Ces coefficients sont également ajustés dans la phase d'établissement d'un upwelling côtier académique le long d'une côte rectiligne infinie. (C) 2001 Ifremer/CNRS/IRD/Éditions scientifiques et médicales Elsevier SAS
\end{abstract}

\begin{abstract}
Data assimilation method by a variational methodology using the adjoint of a 3-D coastal circulation primitive equation model. The optimal control technique using an adjoint method is applied to fit the parameters of a 3-D primitive equation coastal model due to a data assimilation procedure. Two of the main unknowns of coastal modelling operations - open boundary conditions and turbulent mixing processes in stratified conditions - are successively considered. The aim of the boundary control procedure is to identify boundary forcing to ensure the best fit between data and model results by minimising a function that measures model and data discrepancies. A methodology for proceeding from the continuous to the discrete formulation of a control problem is given. The robustness of the method is tested for the typical barotropic case of a semi-enclosed basin Kelvin wave. The data assimilation method of optimal control is then applied to the turbulence closure problem in the framing of coastal oceanic circulation. Numerical simulation of a schematic destruction of an halocline and an upwelling event resulting from a wind stress imposed at the sea surface in a strongly stratified two-layer case are realized. The adjoint method is used to find out an optimal set of
\end{abstract}

* Correspondance et tirés à part.

Adresse e-mail : devenon@ com.univ-mrs.fr (J.L. Devenon).

(C) 2001 Ifremer/CNRS/IRD/Éditions scientifiques et médicales Elsevier SAS. Tous droits réservés 
viscosity and diffusivity coefficients which permits the retrieval of the closest computed simulation to hydrodynamic synthetic observations. (C) 2001 Ifremer/CNRS/IRD/Éditions scientifiques et médicales Elsevier SAS

\section{assimilation de données / circulation côtière 3D / conditions aux limites / modèle adjoint / turbulence}

\section{data assimilation / 3-D coastal circulation / boundary conditions fitting / adjoint method / turbulence}

\section{INTRODUCTION}

Les méthodes d'assimilation de données ont généralement pour objectif de réaliser la synthèse entre plusieurs sources d'information concernant des grandeurs modélisables, évaluées par le calcul d'une part, et observées (directement ou indirectement) par des mesures d'autre part. Cette synthèse s'opère en minimisant un critère d'écart $J$ (on dit aussi critère de « coût ») entre la solution cherchée et les diverses estimations qu'on peut en avoir : observations, résultats de modèle... Ce critère correspond souvent à une norme de l'écart en moyenne quadratique (écart au sens des «moindres carrés »), correspondant à une métrique définie par l'inverse des opérateurs de variance-covariance des estimations dont on dispose: données, première estimation du champ de valeurs à trouver, bruit affectant les relations du modèle...

Ces méthodes ont été tout d'abord mises en œuvre dans les modèles de prédictions météorologiques à des fins opérationnelles. Il s'agissait d'incorporer le plus efficacement possible les observations au fur et à mesure de leur disponibilité en cherchant un champ «analysé » à la fois proche d'une première prédiction et des observations, éventuellement ré-interpolées. Ces approches, pour lesquelles le champ produit n'est pas une solution exacte du modèle, sont dites «faiblement contraintes » Sasaki 1970); les méthodes d'interpolation optimale, ou de filtrage de Kalman, entrent dans cette catégorie.

Le problème posé peut aussi être abordé par l'outil mathématique du contrôle optimal, dont le formalisme a été développé par Lions en 1968 (Lions, 1968) et conduire à une opération d'assimilation de données par méthode d'adjoint. Nous nous sommes pour notre part focalisés sur cette méthode, dite «fortement contrainte » dans laquelle on cherche un contrôle (ou commande) optimal du système, éventuellement sous la forme d'une fonction dépendant de l'espace et du temps, et la solution correspondante du modèle, qui minimise le critère de coût $J$ vis à vis du contrôle. Ainsi posé, le problème est dans le droit fil des méthodes inverses qui consistent à identi- fier les paramètres de fonctionnement d'un modèle (supposé exact) à partir de l'observation de sa solution. Un certain nombre d'études dans différents domaines des sciences de la terre met en évidence l'utilité d'un modèle adjoint servant au calcul du gradient de la fonction de coût et permettant de la minimiser (Brauner et Penel, 1974: Begis et Crepon, 1975; Devenon, 1986) En océanographie côtière, la panoplie des systèmes d'observation actuels (télédétection active ou passive, sondeurs Doppler acoustiques, profileurs) ouvre désormais l'accès à un volume de données sans cesse croissant, offrant de meilleures couvertures et résolutions temporelles et spatiales. Compte tenu de cette évolution technique, la question de la spécification des conditions aux limites et du réglage de certains paramètres des modèles peut être considérée comme relevant d'une problématique plus générale de détermination des paramètres de fonctionnement d'un modèle à partir de l'information apportée par des observations des quantités modélisées. Les études en météorologie utilisant l'outil du calcul variationnel se sont surtout attachées à identifier comme contrôles les conditions initiales (Lewis et Derher, 1985: Talagrand 1991. Compte tenu des problèmes rencontrés pour les phénomènes que nous étudions en océanographie côtière, notamment de la difficulté à spécifier des conditions aux frontières marines du domaine et à paramétriser correctement les phénomènes de mélange verticaux, notre effort s'est principalement porté sur l'identification des conditions aux limites et des coefficients décrivant les effets de la turbulence. Ce sont ces contrôles du modèle que nous chercherons donc à optimiser. Les questions soulevées par l'application de ces méthodes à un code de circulation côtière sont multiples. Elles concernent l'adaptation de la formulation de la méthode de contrôle optimal à un problème de modélisation océanographique contrôlé par ses conditions aux frontières, le passage d'une formulation continue du problème à une formulation discrétisée en différences finies, et les difficultés inhérentes au problème de contrôle d'un coefficient de dissipation distribué au sein de l'emprise du domaine de calcul. On présente dans ce qui suit un 
bilan de synthèse des études effectuées au cours de ces cinq dernières années pour apporter des éléments de réponses à ces questions, chaque problème particulier ayant fait l'objet de publications qui sont citées en référence pour une vision plus complète des techniques numériques mentionnées.

\section{APPLICATION DE LA MÉTHODE DE CONTRÔLE OPTIMAL}

\subsection{Le modèle 3D de circulation côtière}

Le modèle direct ici considéré est un modèle de circulation côtière classique aux équations primitives Leendertse et al, 1977) qui utilise l'hypothèse hydrostatique et les approximations de Boussinesq et de plan $f$. L'état physique du fluide est alors décrit par les équations suivantes pour les variables moyennes:

* Équation de continuité réduite à la condition d'incompressibilité :

$$
\frac{\partial u}{\partial x}+\frac{\partial v}{\partial y}+\frac{\partial w}{\partial z}=0
$$

* Équations du mouvement horizontal :

$$
\begin{aligned}
& \frac{\partial u}{\partial t}+\frac{\partial(u u)}{\partial x}+\frac{\partial(u v)}{\partial y}+\frac{\partial(u w)}{\partial z}-f v+\frac{1}{\rho_{0}} \frac{\partial p}{\partial x}- \\
& \frac{v_{h}}{\rho_{0}}\left(\frac{\partial^{2} u}{\partial x^{2}}+\frac{\partial^{2} u}{\partial y^{2}}\right)-\frac{1}{\rho_{0}} \frac{\partial}{\partial z}\left(v_{t} \frac{\partial u}{\partial z}\right)=0
\end{aligned}
$$

$$
\begin{aligned}
& \frac{\partial u}{\partial t}+\frac{\partial(v u)}{\partial x}+\frac{\partial(v v)}{\partial x}+\frac{\partial(v w)}{\partial x}+f u+\frac{1}{\rho_{0}} \frac{\partial p}{\partial y}- \\
& \frac{v_{h}}{\rho_{0}}\left(\frac{\partial^{2} v}{\partial x^{2}}+\frac{\partial^{2} v}{\partial y^{2}}\right)-\frac{1}{\rho_{0}} \frac{\partial}{\partial z}\left(v_{t} \frac{\partial v}{\partial z}\right)=0
\end{aligned}
$$

* Équation hydrostatique :

$$
\frac{\partial p}{\partial z}=-\rho g
$$

* Équations de transport de la salinité et de la température :

$$
\begin{array}{r}
\frac{\partial S}{\partial t}+\frac{\partial(u S)}{\partial x}+\frac{\partial(v S)}{\partial y}+\frac{\partial(w S)}{\partial z}-v_{h}^{S}\left(\frac{\partial^{2} S}{\partial x^{2}}+\frac{\partial^{2} S}{\partial y^{2}}\right)- \\
\frac{\partial}{\partial z}\left(v_{t}^{S} \frac{\partial S}{\partial z}\right)=0 \\
\frac{\partial S}{\partial t}+\frac{\partial(u T)}{\partial x}+\frac{\partial(v T)}{\partial y}+\frac{\partial(w T)}{\partial z}-v_{h}^{T}\left(\frac{\partial^{2} T}{\partial x^{2}}+\frac{\partial^{2} T}{\partial y^{2}}\right)- \\
\frac{\partial}{\partial z}\left(v_{t}^{T} \frac{\partial T}{\partial z}\right)=0
\end{array}
$$

* Équation d'état :

$$
\rho=\rho_{0}\left(1+\alpha_{s}\left(S-S_{0}\right)+\alpha_{T}\left(T-T_{0}\right)\right)
$$

Dans ces équations, $u, v, w$ sont les trois composantes de la vitesse selon les trois directions d'espace $x$ (vers l'Est), $y$ (vers le Nord) et $z$ (selon la verticale ascendante). $t$ représente le temps, $p$ la pression, $S$ la salinité, $T$ la température et $\rho$ la masse volumique de l'eau de mer. $\rho_{0}, T_{0}$ et $S_{0}$ sont des valeurs de référence constantes respectivement pour la masse volumique, la température et la salinité, $f$ est le paramètre de Coriolis, $g$ l'accélération de la pesanteur, $\alpha_{S}$ le coefficient de contraction saline et $\alpha_{T}$ le coefficient d'expansion thermique, $v_{h}$ et $v_{t}$ les coefficients de viscosité turbulente selon l'horizontale et la verticale, $v_{h}^{S}, v_{t}^{S}, v_{h}^{T}$ et $v_{h}^{T}$ les coefficients de diffusivité turbulente selon l'horizontale et la verticale respectivement pour le sel et la température. Ces coefficients sont à priori variables en espace et en temps.

Une première approche communément considérée, la fermeture turbulente d'ordre zéro, consiste cependant à donner des valeurs constantes aux coefficients de viscosité et de diffusivité turbulente. C'est ce que nous avons adopté dans une première version du modèle utilisée pour l'ajustement des conditions aux frontière ouvertes (section 3.1). Aujourd'hui, il est devenu classique d'utiliser des modèles de turbulence dans lesquels les coefficients de viscosité et de diffusivité turbulente sont fonction de l'état turbulent de l'eau de mer. Parmi ces modèles, on peut choisir une formulation de type longueur de mélange pour tenir compte des effets de stratification. Ainsi dans le modèle $k+L$ (Leendertse et al., 1977) par exemple, le coefficient de viscosité turbulente vertical $v_{t}$ est évalué en utilisant une hypothèse de Kolmogorov étendue prenant en compte les effets de flottabilité :

$$
v_{t}=C_{v} L \sqrt{k} \exp \left(-m R_{i}\right)
$$


où $L$ est une longueur de mélange, $k$ l'énergie cinétique de la turbulence, $m$ et $C \nu$, des constantes positives. $R_{i}$ est le nombre de Richardson turbulent. La longueur de mélange est donnée par une fonction algébrique de la distance au fond $z^{*}$ (Leendertse et al., 1977) :

$$
L=\kappa z^{*} \sqrt{1-\frac{z^{*}}{d}}
$$

où $\kappa$ est la constante de Von Karman et $d$ la hauteur locale de la colonne d'eau.

L'énergie cinétique de la turbulence est alors régie par une équation de transport :

$$
\begin{array}{r}
\frac{\partial k}{\partial t}+\frac{\partial(u k)}{\partial x}+\frac{\partial(v k)}{\partial y}+\frac{\partial(w k)}{\partial z}-v_{h}^{k}\left(\frac{\partial^{2} k}{\partial x^{2}}+\frac{\partial^{2} k}{\partial y^{2}}\right)- \\
\frac{\partial}{\partial z}\left(v_{t}^{k} \frac{\partial k}{\partial z}\right)-P-G+\varepsilon=0
\end{array}
$$

Dans cette équation $P$ est un terme de source par cisaillement, $G$ un terme de source ou puit par effets de flottabilité et $\varepsilon$ un terme de dissipation. $v_{h}^{k}$ est la diffusivité turbulente horizontale pour $k$, usuellement considérée constante, et $v_{t}^{k}$, la diffusivité turbulente verticale pour $k$ supposée proportionnelle à la viscosité turbulente verticale; le coefficient de proportionnalité $\sigma_{k}$ étant souvent appelé le nombre de Prandtl turbulent pour l'énergie cinétique de la turbulence.

\subsection{Le modèle numérique et sa mise en œuvre}

Le modèle numérique multi-niveaux considère la colonne d'eau comme un empilement vertical de couches dont l'épaisseur peut varier suivant la profondeur. Alors que la première couche présente une épaisseur variable pour tenir compte de la surélévation de la surface libre, l'épaisseur des couches suivantes ne présente ni variation horizontale ni variation temporelle. La masse volumique peut être variable à l'intérieur d'une couche. En intégrant sur chaque couche les équations précédentes, on obtient un nouveau jeu d'équations pour les valeurs moyennées sur chaque couche Leredde, 1999. Les transports verticaux de quantité de mouvement à travers les frontières séparant les couches sont calculés en utilisant les composantes de la vitesse horizontale, moyennées sur l'épaisseur des couches, disponibles dans les couches voisines, après que la composante verticale de la vitesse ait été obtenue par intégration de l'équation de continuité entre le fond et le niveau considéré. La grille horizontale de

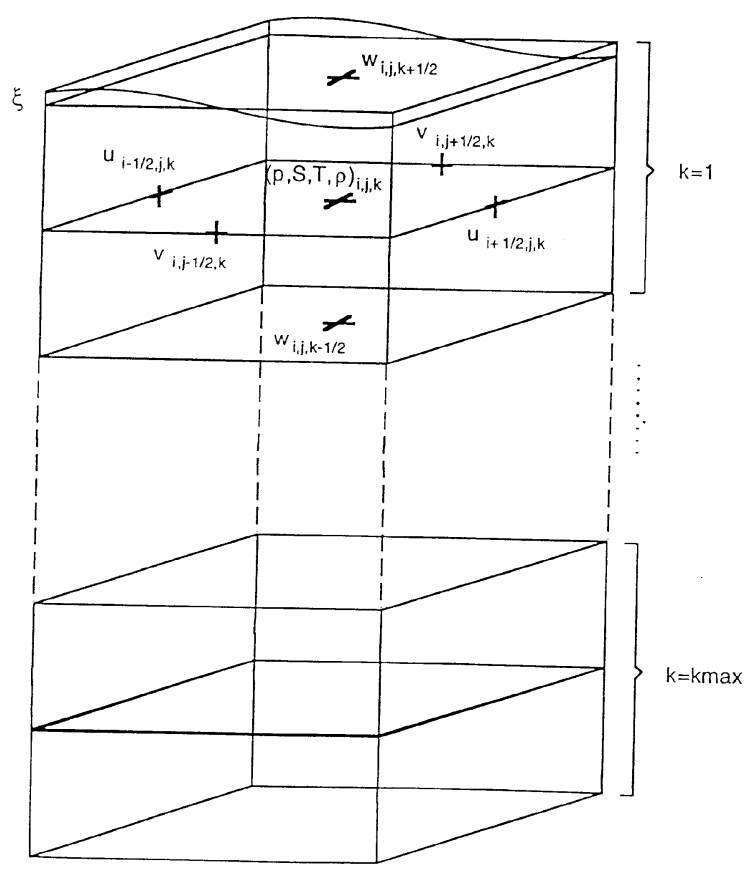

Figure 1. Présentation d'une maille de calcul élémentaire du modèle et localisation des points de calcul des différentes variables.

calcul est une grille décalée de type $\mathrm{C}$ dans la classification d'Arakawa_ef_Lamb (1977) La maille de calcul est le volume qui entoure le point où sont évaluées les quantités scalaires (pression $p$, salinité $S$, température $T$, masse volumique $\rho$ ). Les points en lesquels sont évalués les composantes de vitesse $u$, $v$, et $w$ sont situés au centre des facettes de ce volume (Ggure - d $)$. Cela simplifie le calcul des flux d'advection-diffusion d'une quantité scalaire à travers les facettes limitant la maille de calcul ainsi que la spécification numérique des conditions aux limites. Par ailleurs, la version discrétisée des équations aux dérivées partielles exprimée sur la grille décentrée de type $\mathrm{C}$ demande que soient effectuées des moyennes spatiales pour disposer de toutes les variables nécessaires aux endroits requis. Comme ces moyennes impliquent automatiquement des quantités appartenant à des mailles voisines, les calculs menés au voisinage des frontières mettent en jeu des valeurs aux limites.

\subsection{Formulation de la méthode de contrôle optimal}

L'objectif d'une méthode variationnelle de contrôle optimal est de trouver le meilleur jeu de paramètres du 
modèle pour produire une simulation qui soit la plus proche possible des valeurs observées (Devenon. 1986. 1990:_Le Dimet et Talagrand. 1986: Bennett_ 1992). On en montre ici un exemple d'application lorsque le paramètre de viscosité turbulente vertical est considéré comme la fonction à optimiser. On pose donc $J\left(v_{t}\right)=\left\|Y-Y^{\mathrm{obs}}\right\|^{2}$, où l'état physique du système est décrit par le vecteur d'état: $Y=(u, v, w, p, S, T, \rho)$ et où $Y^{\text {obs }}$ désigne le vecteur des observations $Y^{\text {obs }}=(\hat{u}, \hat{v}, \hat{w}, \hat{p}, \hat{S}, \dddot{T}, \hat{\rho}) .\|\cdot\|$ désigne la norme au sens $L^{2}$, éventuellement pondérée par l'inverse des opérateurs de covariance des observations. Envisageons le cas simple d'observations dont les erreurs sont statistiquement indépendantes, et suivent des lois gaussiennes de variances identiques ; $S_{i} \delta v_{t}$ désigne un «petit » accroissement du paramètre de contrôle $v_{t}$, on montre aisément que

$$
d J=\left(Y-Y^{o b s}, \frac{\partial Y}{\partial v_{t}} \delta v_{t}\right)_{L^{2}}
$$

l'état adjoint $Y^{*}=\left(u^{*}, v^{*}, w^{*}, p^{*}, S^{*}, T^{*}, \rho^{*}\right)$ est alors introduit pour faire apparaître $d J$ comme une forme linéaire de $\delta v_{t}$.

En rappelant que $A^{*}$ est l'opérateur adjoint de $A$ s'il vérifie $\left(A^{*} X, Z\right)_{L^{2}}=(X, A Z)_{L^{2}}$, on définit l'état adjoint $Y^{*}$ par la relation :

$$
\left(\frac{\partial F}{\partial Y}\right)^{*} Y^{*}=Y-Y^{o b s}
$$

Alors :

$$
d J=\left(Y^{*}, \frac{\partial F \partial Y}{\partial Y \partial v_{t}} \delta v_{t}\right)_{L^{2}}
$$

Le modèle hydrodynamique établi par ailleurs un lien implicite entre $Y$ et $v_{t}: F\left(Y, v_{t}\right)=0$, de sorte que

$$
\frac{\partial F}{\partial v_{t}} \delta v_{t}+\frac{\partial F \partial Y}{\partial Y \partial v_{t}} \delta v_{t}=0
$$

et par conséquent :

$d J=\left(Y^{*}, \frac{\partial F}{\partial v_{t}} \delta v_{t}\right)_{L^{2}}=\left(-\left(\frac{\partial F}{\partial v_{t}}\right)^{*} Y^{*}, \delta v_{t}\right)_{L^{2}}=\left(\nabla J, \delta v_{t}\right)_{L^{2}}$

On a donc :

$$
\nabla J=-\left(\frac{\partial F}{\partial v_{t}}\right)^{*} Y^{*}
$$

Le gradient de $J$ est donc calculable par l'équation (2) dès que l'état adjoint $Y^{*}$, solution du modèle adjoint du modèle linéaire tangent forcé par l'écart aux observations (cf. équation (1)), est connu. L'obtention de ce gradient permet de mettre en œuvre un algorithme de minimisation de $J$ par une méthode de descente.

Tous calculs faits, on montre, lorsque les variances d'erreurs affectant les observations sont des fonctions constantes de l'espace et du temps, que la forme des équations adjointes est la suivante :

$$
\begin{gathered}
\frac{\partial u^{*}}{\partial t}+2 u \frac{\partial u}{\partial x}+v\left(\frac{\partial u^{*}}{\partial y}+\frac{\partial v^{*}}{\partial x}\right)+w \frac{\partial u^{*}}{\partial z}-f v^{*}+S \frac{\partial S^{*}}{\partial x}+ \\
T \frac{\partial T^{*}}{\partial x}+\frac{v_{h}}{\rho_{0}}\left(\frac{\partial^{2} u^{*}}{\partial x^{2}}+\frac{\partial^{2} u^{*}}{\partial y^{2}}\right)+\frac{1}{\rho_{0}} \frac{\partial}{\partial z}\left(v_{t} \frac{\partial u^{*}}{\partial z}\right)=\frac{u-\hat{u}}{\sigma_{\hat{u}}^{2}} \\
\frac{\partial v^{*}}{\partial t}+2 v \frac{\partial v^{*}}{\partial y}+u\left(\frac{\partial v^{*}}{\partial x}+\frac{\partial u^{*}}{\partial y}\right)+w \frac{\partial v^{*}}{\partial z}+f u^{*}+S \frac{\partial S^{*}}{\partial y}+ \\
T \frac{\partial T^{*}}{\partial y}+\frac{v_{h}}{\rho_{0}}\left(\frac{\partial^{2} v^{*}}{\partial x^{2}}+\frac{\partial^{2} v^{*}}{\partial y^{2}}\right)+\frac{1}{\rho_{0}} \frac{\partial}{\partial z}\left(v_{t} \frac{\partial v^{*}}{\partial z}\right)=\frac{v-\hat{v}}{\sigma_{\hat{v}}^{2}} \\
\frac{\partial p^{*}}{\partial z}-\frac{1}{\rho_{0}}\left(\frac{\partial u^{*}}{\partial x}+\frac{\partial y^{*}}{\partial y}\right)=\frac{p-\hat{p}}{\sigma_{\hat{p}}^{2}} \\
\frac{\partial w^{*}}{\partial z}+u \frac{\partial u^{*}}{\partial z}+v \frac{\partial v^{*}}{\partial z}+S \frac{\partial S^{*}}{\partial z}+T \frac{\partial T^{*}}{\partial z}=\frac{w-\hat{w}}{\sigma_{\hat{w}}^{2}} ; \\
\rho^{*}+g p^{*}=\frac{\hat{\rho}-\rho}{\sigma_{\hat{\rho}}^{2}} \\
\frac{\partial S^{*}}{\partial t}+u \frac{\partial S^{*}}{\partial x}+v \frac{\partial S^{*}}{\partial y}+w \frac{\partial S^{*}}{\partial z}+\rho_{0} \alpha_{S} \rho^{*}+ \\
v_{h}^{S}\left(\frac{\partial^{2} S^{*}}{\partial x^{2}}+\frac{\partial^{2} S^{*}}{\partial y^{2}}\right)+\frac{\partial}{\partial z}\left(v_{t}^{S} \frac{\partial S^{*}}{\partial z}\right)=\frac{S-\hat{S}}{\sigma_{\hat{S}}^{2}}
\end{gathered}
$$

$$
\begin{aligned}
\frac{\partial T^{*}}{\partial t}+u \frac{\partial T^{*}}{\partial x}+ & v \frac{\partial T^{*}}{\partial y}+w \frac{\partial T^{*}}{\partial z}+\rho_{0} \alpha_{T} \rho^{*}+ \\
& v_{h}^{T}\left(\frac{\partial^{2} T^{*}}{\partial x^{2}}+\frac{\partial^{2} T^{*}}{\partial y^{2}}\right)+\frac{\partial}{\partial z}\left(v_{t}^{T} \frac{\partial T^{*}}{\partial z}\right)=\frac{T-\hat{T}}{\sigma_{\hat{S}}^{2}}
\end{aligned}
$$

$\sigma_{\Theta}^{2}$ est la variance de l'erreur de l'observation $\hat{\Theta}$ correspondant à la variable $\Theta$. Le détail des calculs conduisant à ce système d'équations est donné dans les références Leredde et al., 1998, 1999: Leredde, 1999). Le gradient de la fonction de coût $J$ s'exprime alors de manière simple en fonction de l'état adjoint $Y^{*}$ Lellouche.1995. Leredde, 1999) 


\subsection{Passage d'une formulation continue à une formulation discrète}

Depuis les premières applications du contrôle optimal pour les approches variationnelles en océanographie et en météorologie, un grand nombre d'auteurs ont relevé la difficulté de passer d'une version continue de l'application de la méthode à une version discrétisée en préservant toutes les propriétés mathématiques. Ceci fut à l'origine d'un certain nombre de travaux pour obtenir le code adjoint d'une façon plus ou moins automatique en opérant directement sur les instructions du code de calcul. En adoptant une autre démarche, nous avons décidé de porter notre attention sur les difficultés qui apparaissent lors de l'étape de discrétisation. Le principal problème est que le schéma de discrétisation couramment utilisé pour le modèle direct ne préserve pas les propriétés de la formulation continue de la méthode de contrôle optimal. En conséquence, la propriété importante, pour les deux modèles, d'être adjoints l'un de l'autre, est perdue au cours de l'opération. Un adjoint discrétisé erroné conduit à une mauvaise formulation discrétisée du gradient de la fonctionnelle de coût. En fait, des relations de compatibilité sont indispensables pour discrétiser correctement les dérivées spatiales et temporelles au voisinage des frontières. Le problème est alors d'établir des conditions aux frontières consistantes pour le modèle adjoint à partir de celles du modèle direct. Pour tenir compte de cette difficulté, des discrétisations adéquates sont proposées pour les modèles direct et adjoint. L'étude d'un modèle d'advection-diffusion - en l'occurrence le modèle unidimensionnel gouverné par l'équation de Burger - nous a permis (Lellowche_ef_al, 1994) de dégager des règles de discrétisation conduisant à une évaluation exacte du gradient de la fonction de coût fondée sur une approche matricielle. Notamment, le choix classique d'une norme $L_{2}$ pour mesurer les écarts entre la solution du modèle et les observations nous a amené à proscrire l'utilisation de valeurs de frontière dans tous les termes discrétisés excepté celui de diffusion. Cette discrétisation présente aussi la bonne propriété de réduire les instabilités numériques qui apparaissent lors de la prise en compte des conditions aux limites dans les termes non-linéaires d'advection car ces dernières n'interviennent plus que dans un Laplacien. Ces règles ont été étendues au modèle 3D de circulation côtière Lellouche et al., 1998). Sans précautions particulières, les moyennes spatiales effectuées sur la grille $\mathrm{C}$ auraient utilisé les valeurs aux nœuds frontière dans les calculs effectués pour les mailles
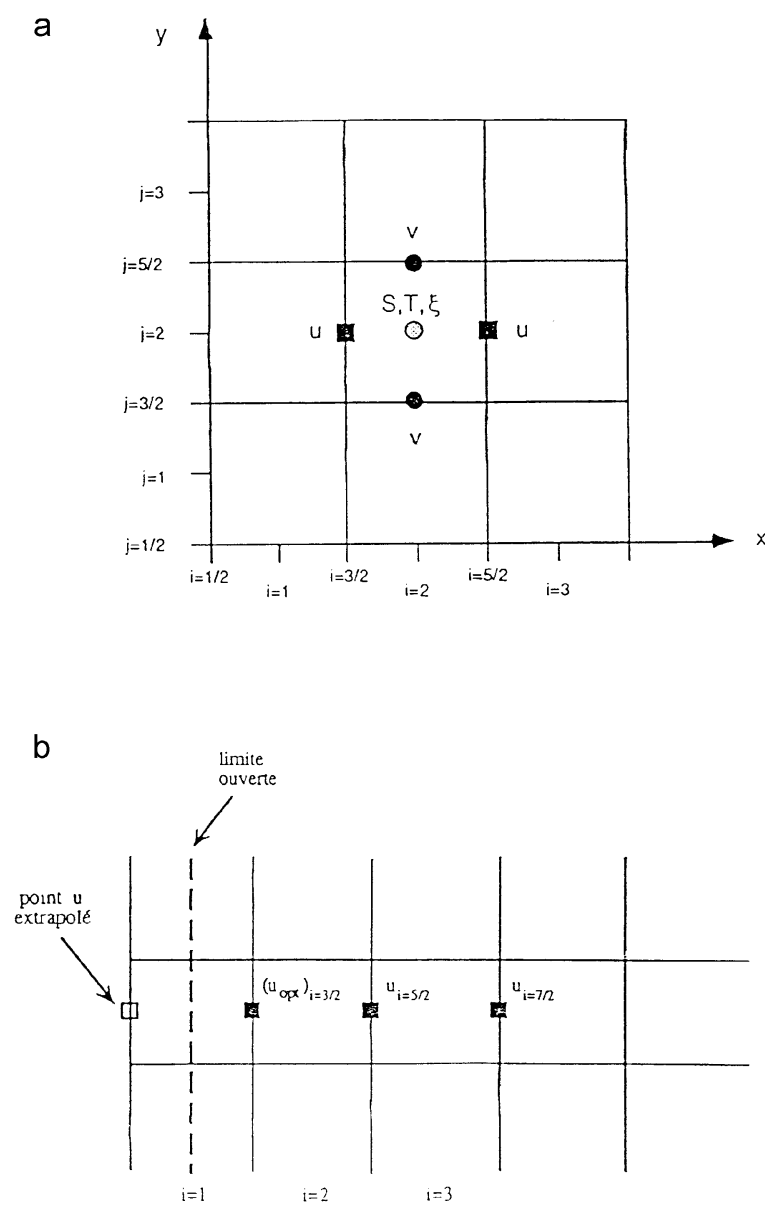

Figure 2. a. Décentrement de la grille de calcul de type $\mathrm{C}$ dans le plan horizontal. b. Extrapolation des variables à une maille et demi au voisinage de la frontière ouverte.

situées au voisinage de celle-ci. C'est précisément ce qui doit être évité pour les termes autres que ceux de diffusion turbulente. Pour pallier à cet inconvénient, nous avons utilisé une extrapolation linéaire à l'échelle d'une maille et demie au voisinage des frontières ouvertes (figure 2a, $2 b$ ).

\section{RÉSULTATS DES EXPÉRIENCES NUMÉRIQUES D'AJUSTEMENT}

\subsection{Identification des conditions aux limites : onde de Kelvin en bassin semi-fermé}

Le code assimilateur a été testé dans le cas simple d'un écoulement océanique associé à une onde longue de 
gravité barotrope essentiellement forcé par les conditions imposées aux limites ouvertes. L'objectif est ici de tester la capacité de la procédure d'assimilation à identifier les conditions aux limites ouvertes dans un cas typique de situation côtière. Pour ce faire, nous avons considéré un bassin semi-fermé parallélépipédique de profondeur constante $H$, situé à la latitude de $45^{\circ} \mathrm{N}$ (figure 3). L'eau de mer est supposée de masse volumique constante et seule la frontière ouest du bassin est ouverte. La largeur du bassin est de l'ordre du double du rayon externe de déformation de Rossby $R$. La circulation, résultante de la superposition sur la frontière ouest d'une onde de Kelvin entrante au sud et sortante au nord, et se propageant respectivement le long des côtes sud et nord du bassin dans des directions opposées, est simulée à l'aide du modèle direct. La longueur d'onde commune des ondes entrante et sortante est de l'ordre de la moitié de la longueur $L$ du bassin. Cette solution de référence, correspondant à un jeu de conditions aux limites connu, joue le rôle des observations à assimiler. On teste donc l'aptitude de la méthode à retrouver le jeu correct de conditions aux limites ayant servi à construire ces observations «idéales » vérifiant exactement les équations du modèle. Pour attester de la validité de la méthode, l'exactitude du gradient obtenu est contrôlée par la vérification d'une formule de Taylor (Talagrand et Courtier, 1987) figurd 4b). Le test est mené sur des expressions adimensionnalisées de sorte que l'accroissement $h$ du contrôle est également une variable sans dimension. Le point initial de la procédure de minimisation correspond à une perturbation «raisonnable» des conditions de frontière ouverte ayant servies à simuler le champ des observations. La fonction de coût est alors minimisée sans aucune contrainte. On suppose, de façon idéale, que des observations sont disponibles en chaque nœud de grille et chaque instant. L'efficacité de la méthode à retrouver les conditions de frontière ouverte est effectivement vérifiée. Un jeu de contrôles optimaux est obtenu en imposant un critère de convergence sur la valeur de la norme du gradient de $J$. Ici, un critère de 0.1 conduit à l'arrêt de la minimisation au terme de 55 itérations (Goure 4d) d'un algorithme de quasi-Newton Gilhert et Lemarechal 1989). Les valeurs de $J$ et de son gradient son représentées à titre d'exemple jusqu'à environ 68 itérations. La solution du modèle direct correspondant au jeu de conditions de frontière associé au début de la minimisation a été considérablement améliorée de sorte que la topographie observée de la surface libre a été retrouvée avec une grande précision. Les champs de surélévation, observés et

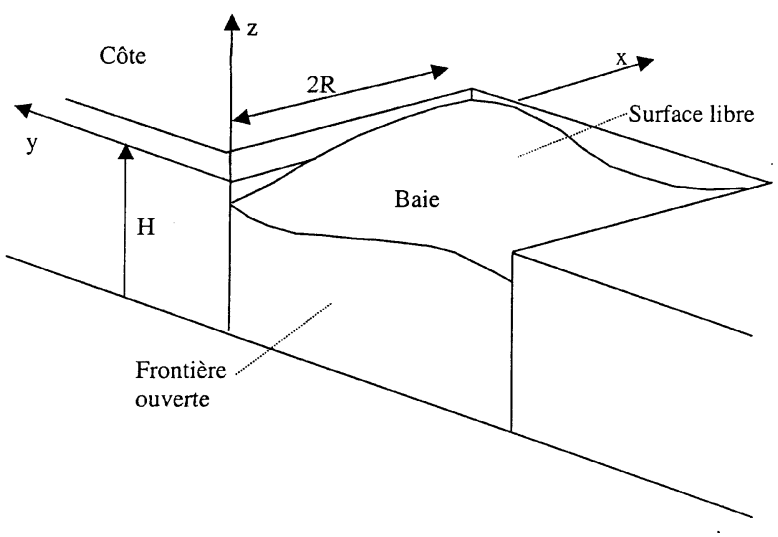

Figure 3. Schéma de principe de l'expérience d'identification des conditions aux limites dans le cas d'une seiche de Kelvin dans une baie semi-ouverte.

correspondant au contrôle optimal, sont indiscernables figure 5d-c). Ceci met en évidence que dans ce cas d'observation totale du système, un contrôle par les conditions aux frontières ouvertes peut être obtenu avec précision. Les observations «simulées » sont parfaitement retrouvées après l'opération d'assimilation. D'autres tests incluant du bruit et des lacunes dans les observations simulées pourront être trouvés ailleurs LelLuche, 1995: Lellowche et al, 1998).

\subsection{Identification de la viscosité turbulente}

Dans les modèles de circulation océanique, le système formé par les équations de continuité et de conservation de quantité de mouvement, de chaleur et de sel est un système ouvert. Des équations supplémentaires reliant les tensions de cisaillement au champ de vitesses moyennes, et les flux diffusifs aux gradients de température et de salinité moyennes doivent être introduites. La fermeture du système d'équations est classiquement réalisée à l'aide de l'hypothèse de viscosité Newtonienne. Par le passé, des valeurs constantes (avec des valeurs horizontale et verticale différentes) ou des variations empiriques, permettant une prise en compte plus réaliste des effets non-linéaires, ont été adoptées pour la viscosité turbulente. Lorsque la viscosité et les diffusivités turbulentes verticales ont une influence significative sur les résultats de simulation, notamment dans les situations de mers stratifiées, celles-ci doivent être spécifiées avec plus de précision. Les capacités des calculateurs actuels autori 


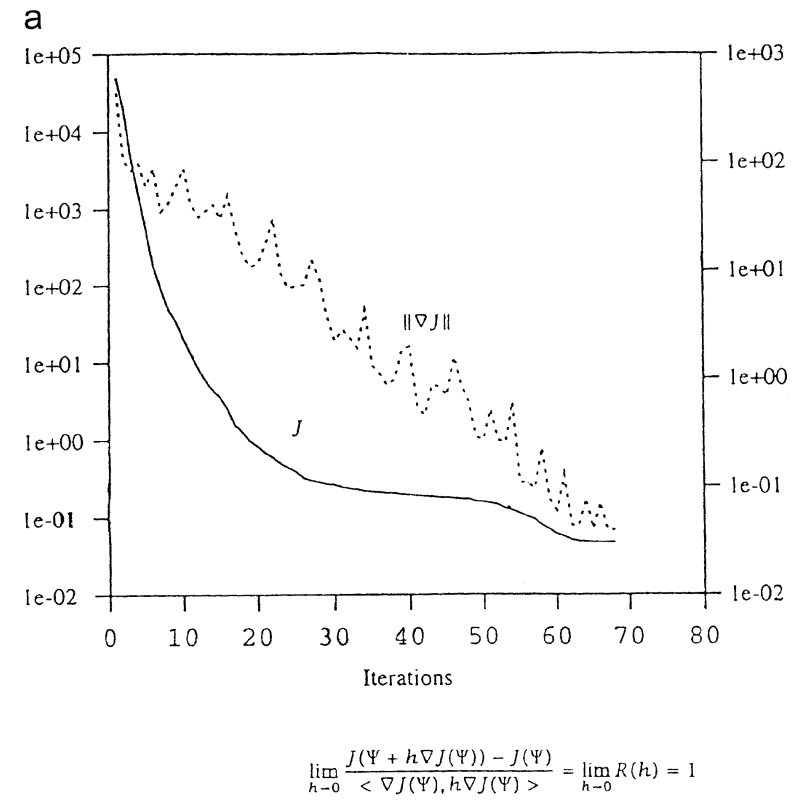

b

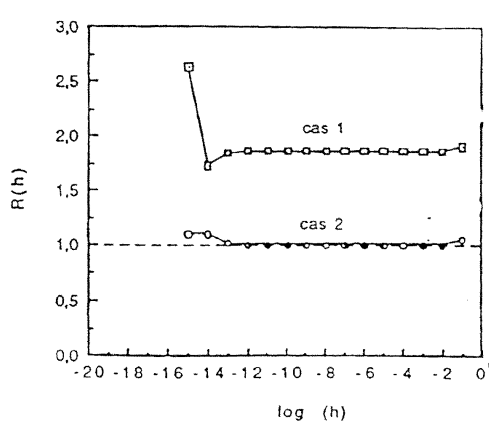

Figure 4. a. Évolution de la fonction de coût et de son gradient au cours de la procédure d'optimisation. b. Mise en œuvre d'un test de validité du gradient par une formule de Taylor; le cas 1 est celui d'une discrétisation de l'adjoint déduite sans précaution de celle du modèle direct, le cas 2 étant celui d'une discrétisation correcte du modèle et de l'adjoint.

sent l'utilisation de valeurs locales de quantités descriptives de la turbulence pour déterminer ces coefficients de diffusion. De telles approches font appel à des équations de transport additionnelles. Les modèles actuels, de plus en plus perfectionnés en ce qui concerne la modélisation numérique de la turbulence, dépendent d'un grand nombre de paramètres à calibrer à partir de résultats expérimentaux disponibles en quantité limitée. De plus, il subsiste de nombreuses incertitudes qui ne peuvent être prises en compte par ces modèles de turbulence. Comme approche alternative, une méthode inverse est ici propo-
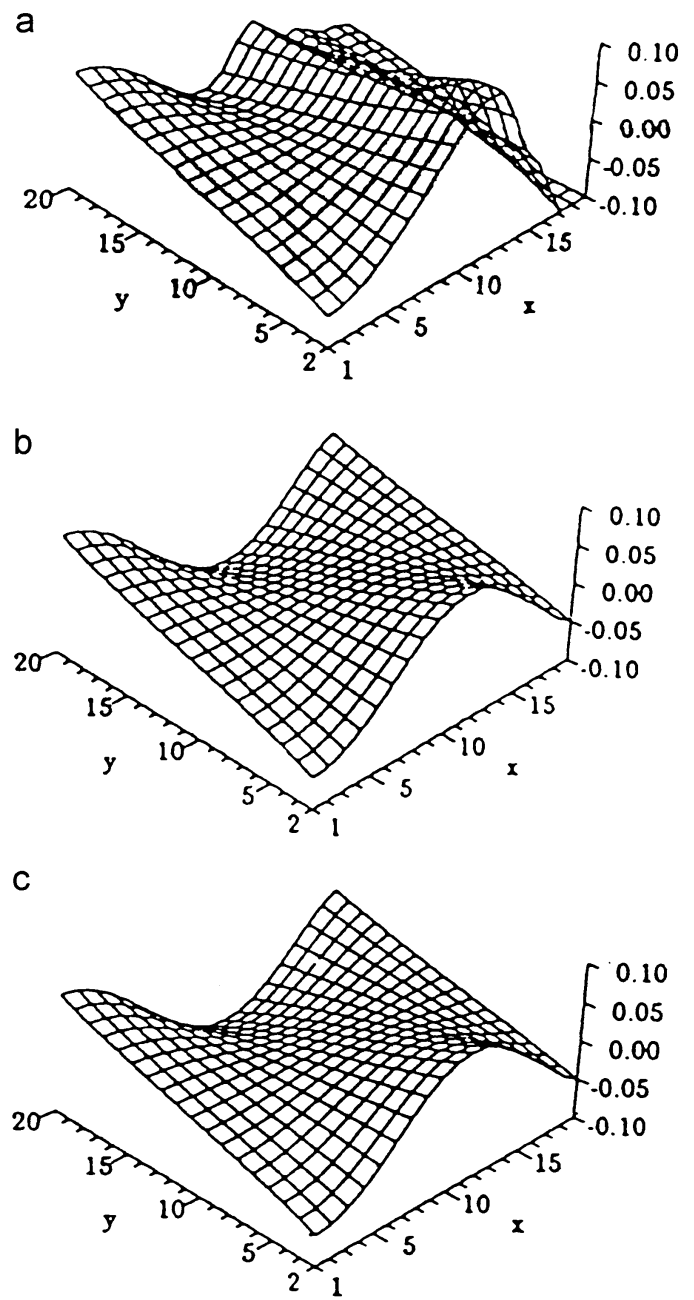

Figure 5. Allure des champs de surélévation de la surface marine dans le cas de la seiche de Kelvin : a. solution du modèle au début de la procédure de minimisation, b. observations simulées à retrouver, $\mathbf{c}$. solution du modèle correspondant aux conditions aux limites optimales.

sée. La viscosité et les diffusivités turbulentes sont identifiées à partir de données océanographiques. La méthode de contrôle optimal est ici développée dans le contexte plus général de l'optimisation d'une viscosité turbulente susceptible de varier dans les trois directions de l'espace et dans le temps. Le grand nombre de paramètres à optimiser requiert un nombre au moins aussi grand de données à assimiler. Nous avons donc choisi de construire des observations synthétiques à l'aide d'un modèle de turbulence d'ordre supérieur. Le modèle retenu est le modèle multi-niveaux $k+L$ (cf. section 2.1) utilisant une seule équation de transport 
additionnelle pour l'énergie cinétique de la turbulence $k$ et une formulation algébrique pour la longueur de mélange $L$. Cela permet de construire un jeu d'observations et un jeu associé de valeurs de viscosité turbulente à retrouver par méthode inverse.

\subsubsection{Situation $1 D$ : rupture d'une halocline}

En sus du coût numérique, il est difficile de résoudre des problèmes d'optimisation pour lesquels, après discrétisation, la taille du vecteur des contrôles égale le nombre de points de grille et de pas de temps. Par suite, les premières applications de notre modèle assimilateur concernent des processus océanographiques relativement simples permettant le traitement numérique avec une dimension acceptable. Aussi, en mer infinie, un épisode de mélange sous l'effet du vent d'une colonne d'eau uniquement stratifiée en salinité, induisant des oscillations d'inertie, est simulé par le modèle 3D multi-niveaux $k+L$. Ce processus est en fait $1 \mathrm{D}$ vertical. L'opération d'assimilation la plus élémentaire consiste à considérer que toutes les données assimilées $u, v, S$ sont exemptes d'erreurs. Les viscosités turbulentes optimisées conduisent à des champs analysés $u, v, S$ indiscernables des observations (figure $)$. Ces résultats montrent la faisabilité de la méthode sans introduire dans la fonction de coût un terme de rappel à une ébauche préalable des contrôles. Cependant, il faut rappeler qu'une telle qualité d'ajustement entre la solution du modèle et les données n'est rendue possible qu'en raison de l'obtention du jeu de données simulées à partir du modèle de turbulence $k+L$ complet, et donc par conséquent totalement compatible avec les contraintes d'optimisation. Ceci ne pourra être le cas pour des données in situ réelles. Néanmoins, on peut remarquer que le jeu optimal de valeurs de viscosité est par endroits légèrement différent de celui produit par le modèle $k+L$ lors de la construction des observations. Ainsi, même lorsqu'ils diffèrent localement, deux champs de viscosité peuvent-ils conduire à des solutions hydrodynamiques indiscernables au sens de la norme en moyenne quadratique adoptée pour $J$ et de la précision de la machine ? La procédure d'optimisation que nous avons menée produit un champ de viscosité possédant un degré de liberté par noud de grille spatiale et par pas de temps, tandis que le modèle de turbulence $k+L$ contraint beaucoup plus la cohérence spatiale et temporelle des valeurs de viscosité du fait du faible nombre de constantes numériques dont il dépend et des équations qu'il utilise. De plus, la viscosité turbulente n'apparaît que dans les termes de diffusion des équations de quantité de mouve- ment, de sorte que son influence sur la solution du modèle est pratiquement inexistante dans les régions de très faibles gradients spatiaux des champs de vitesse. Ceci pose clairement le problème du contrôle de la solution du modèle en agissant sur des coefficients qui n'ont pas partout une influence significative.

\subsubsection{Cas bidimensionnel d'un upwelling côtier}

La taille du vecteur contrôle était déjà importante dans le cas à une dimension d'espace précédent, ce qui implique un coût numérique élevé, une importante capacité de stockage en mémoire, et une convergence assez lente de la méthode. Ce coût est augmenté par l'utilisation des valeurs de la solution du modèle direct sur tout l'intervalle temporel d'assimilation pour forcer le modèle adjoint. Les variables correspondantes doivent donc être stockées en mémoire lors du calcul direct, puis récupérées dans l'ordre inverse lors du fonctionnement de l'adjoint. C'est la raison pour laquelle nous limitons ici volontairement la dépendance du champ de viscosité à deux dimensions d'espace et le considérons comme indépendant du temps.

Pour des raisons similaires, un modèle simplifié d'océan côtier et de forçage par le vent avec une grille de calcul à un petit nombre de mailles a été utilisé ici. L'intégration des équations du modèle n'est également envisagée que sur une durée assez courte. En fait, c'est la distribution verticale de température qui engendre la stratification. Le problème de l'optimisation d'un coefficient de viscosité dépendant uniquement de deux coordonnées d'espace est illustré à partir d'un épisode de mise en place d'un upwelling côtier. Une côte rectiligne, où l'on impose une condition d'imperméabilité, limite le domaine de calcul à l'Ouest, tandis qu'un vent du Sud constant en force et en direction souffle à $5 \mathrm{~m} \cdot \mathrm{s}^{-1}$ (figure - En supposant le domaine illimité au Nord comme au Sud, on peut faire l'hypothèse que la solution ne varie pas avec la coordonnée $y$. Ce domaine situé à la latitude de $45^{\circ} \mathrm{N}$, de profondeur constante $(40 \mathrm{~m})$ et s'étendant à $40 \mathrm{~km}$ au large, est discrétisé avec une maille de $2 \times 2 \mathrm{~km}$ à l'horizontale et de $2 \mathrm{~m}$ sur la verticale. La simulation, d'une durée de $48 \mathrm{~h}$ avec un pas temporel constant de $50 \mathrm{~s}$, est réalisée à partir d'un état initial de repos, avec une distribution verticale de température obéissant à une loi de décroissance linéaire à partir d'une température de surface de $25^{\circ} \mathrm{C}$. Les observations à assimiler sont construites à partir d'un champ de viscosité stationnaire, bidimensionnel (plan vertical), correspondant à la solu 

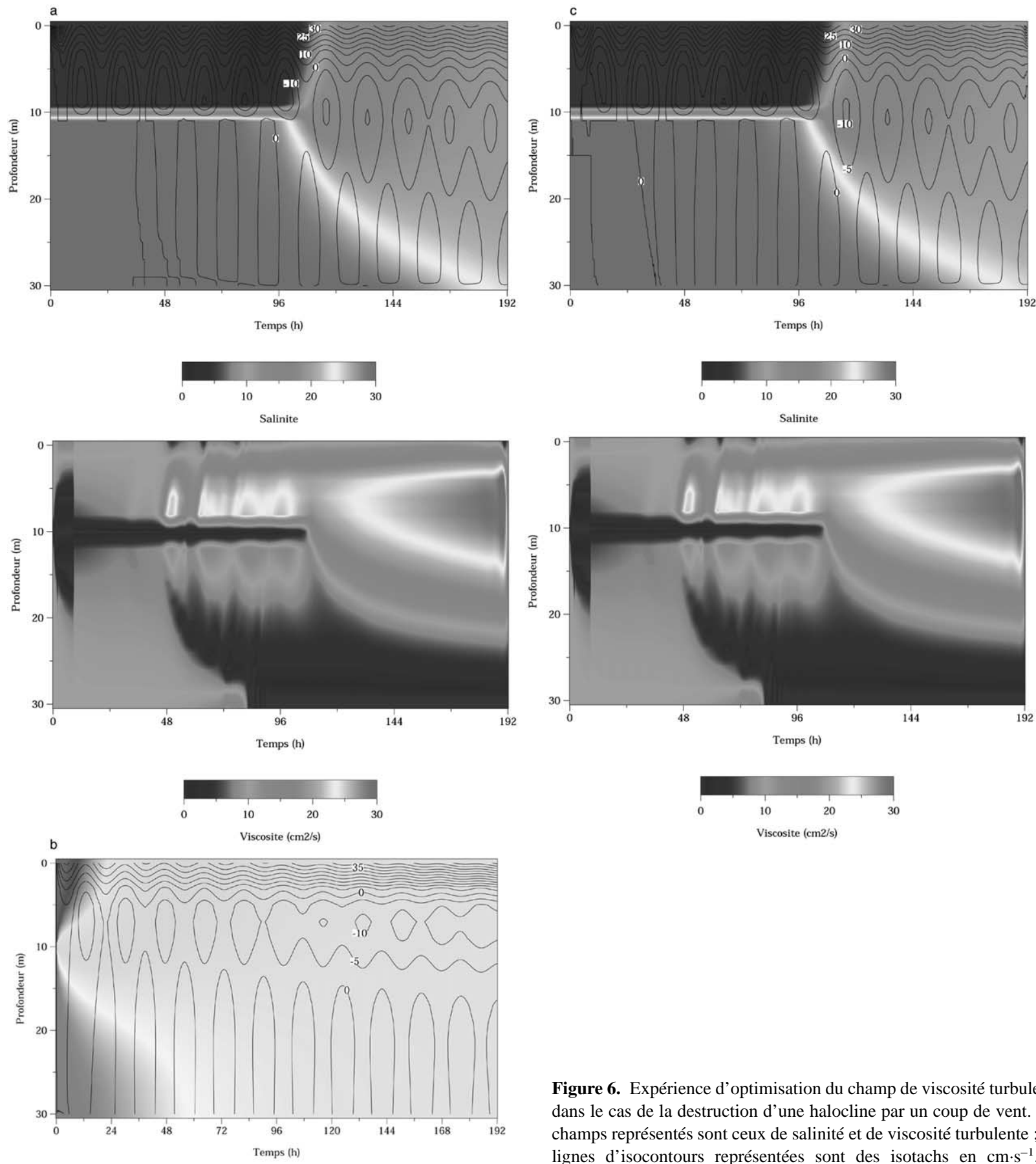

Figure 6. Expérience d'optimisation du champ de viscosité turbulente dans le cas de la destruction d'une halocline par un coup de vent. Les champs représentés sont ceux de salinité et de viscosité turbulente ; les lignes d'isocontours représentées sont des isotachs en $\mathrm{cm} \cdot \mathrm{s}^{-1}$. a. Observations simulées au moyen du modèle $k+L$. b. Solution du modèle, obtenue pour une viscosité turbulente constante de $10 \mathrm{~cm}^{2} \cdot \mathrm{s}^{-1}$, au début de la procédure d'optimisation. c. Solution obtenue après optimisation du champ de viscosité turbulente. 


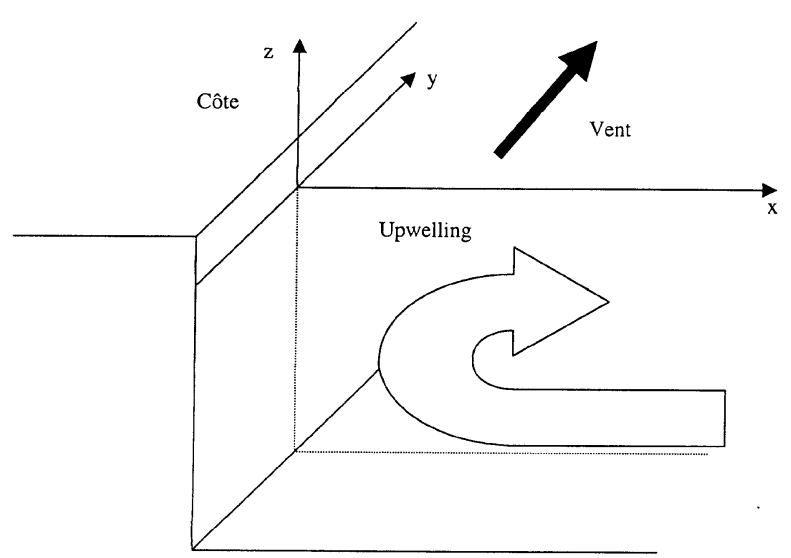

Figure 7. Schéma de principe de l'expérience d'identification du coefficient de viscosité turbulente pour le cas de l'upwelling côtier.

tion fournie par le modèle $k+L$ au bout de $48 \mathrm{~h}$ de simulation. Pour des durées de simulation supérieures, on constate en effet que le champ de viscosité n'évolue pratiquement plus et c'est pourquoi il est choisi comme contrôle stationnaire à retrouver. Les autres variables de contrôle possibles du modèle, telles par exemple les conditions initiales ou aux limites, sont supposées parfaitement connues dans ce problème. L'amorce de la procédure d'optimisation est effectuée à partir d'une solution du modèle correspondant à une valeur uniforme arbitraire de viscosité turbulente égale à $10 \mathrm{~cm}^{2} \cdot \mathrm{s}^{-1}$. Pour rendre le test plus probant au regard de la disponibilité des données dans une situation réelle, seules les données de température sont assimilées. En particulier, le champ de vitesse est supposé inconnu. Comme la viscosité turbulente est supposée constante en fonction du temps, l'observation du champ de température à un seul instant suffit à identifier le jeu de contrôles. Dans ce problème d'optimisation idéalisé, on a choisi de ne considérer qu'une observation exacte du champ de température à $t=48 \mathrm{~h}$. La méthode produit, par inversion de ces données partielles, un champ de viscosité turbulente conduisant à une solution hydrodynamique relativement précise en vitesse et en température sur toute la durée d'établissement de l'upwelling (figure $8 a$, $b$ ).

\section{DISCUSSION ET CONCLUSION}

Les études menées en assimilation de données par méthode variationnelle utilisant l'adjoint ont permis de progresser vers l'application de cette méthode à un code
3D de circulation côtière. La prise en compte d'une discrétisation correcte du code direct et du code adjoint pour obtenir une expression correcte du gradient de la fonction de coût pourrait être par exemple de nature à donner éventuellement des indications sur la démarche à adopter, pour faciliter (ou rendre plus naturel) les écritures de codes adjoint par d'autres méthodes. La compréhension de l'origine de ces difficultés mériterait sans doute un examen mathématique plus approfondi (Bosseur. 1998) et le passage discret/continu reste donc un débat relevant d'études spécifiques Sirkes et Tziperman. 1997), bien que la tendance actuelle pour résoudre le problème en pratique ait consisté à faire un usage assez systématique de technique d'obtention automatique d'adjoint Talagrand, 1991)

En vue des applications pratiques, l'opération d'identification des conditions aux limites peut aussi constituer une alternative intéressante à la spécification classique des conditions de frontière. En modélisation côtière, celle-ci se heurte traditionnellement à la double difficulté de laisser sortir du domaine les mouvements de haute fréquence spatiale ou temporelle, ou la circulation engendrée par le vent local, tout en laissant pénétrer l'influence de la circulation de grande ou de moyenne échelle, ce qui a donné naissance à un grand nombre de techniques souvent semi-empiriques (conditions de radiation, couche éponge, relaxation vers la circulation générale à proximité des frontières...). D'une manière plus générale, l'interfaçage du modèle de circulation côtière avec des modèles de plus grande emprise utilisant une physique différente et des schémas de discrétisation différents semble pouvoir être une voie d'application «naturelle » de telles méthodes d'optimisation, dans laquelle l'interaction entre les modèles n'est plus limitée à la seule frontière, mais est réalisée sur une zone plus étendue.

L'optimisation des coefficients de turbulence dans les cas fortement stratifiés ou de brusque disparition de stratification répond quant à elle à une problématique plus spécifiquement estuarienne ou de zone de dilution fluviale - Region of Freshwater influence ou RoFi au sens de Simpson (Simpson et al., 1993). Une première étape a été franchie en réussissant à optimiser des champs de viscosité turbulente selon la verticale. Cette technique, si elle peut être mise en œuvre sans conduire à des problèmes trop sous-déterminés ou trop volumineux, peut rendre des services utiles dans la pratique, et permettre d'avoir une meilleure connaissance des réactions d'un modèle à son paramétrage des mélanges verticaux. La démarche natu 
a
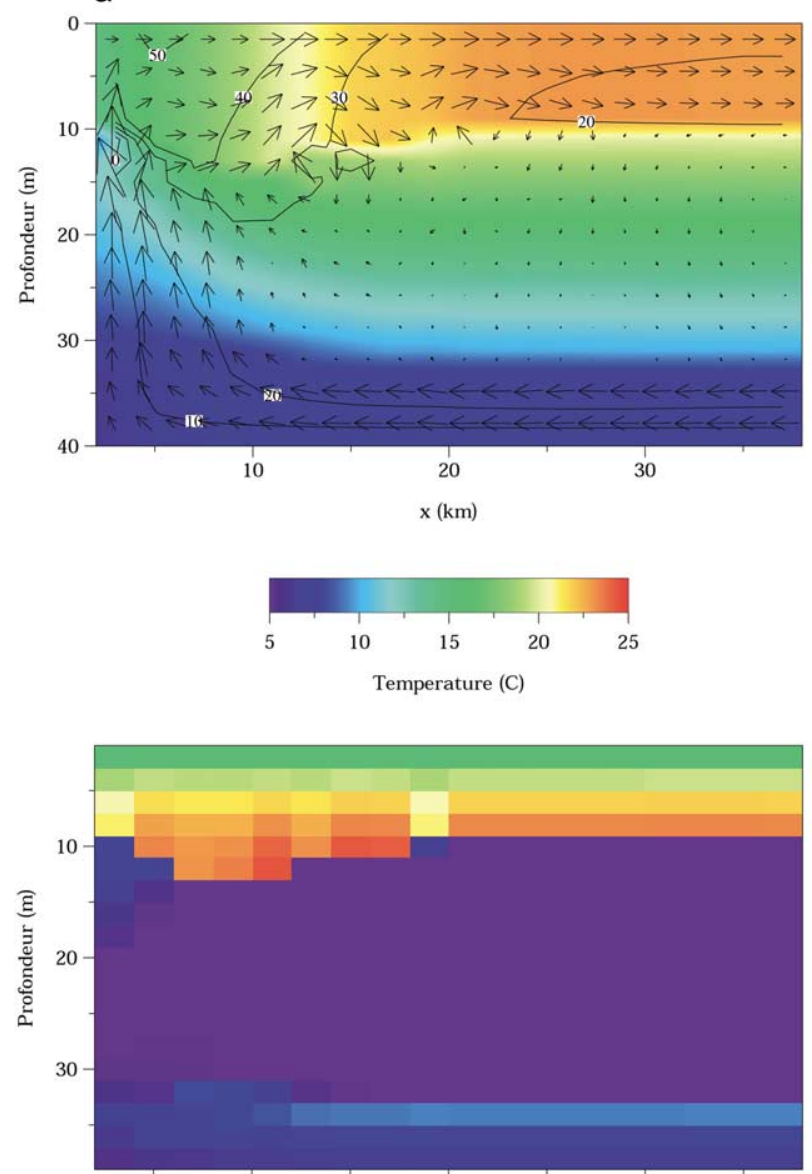

10

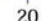

$\mathrm{x}(\mathrm{km})$

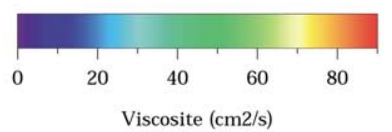

b
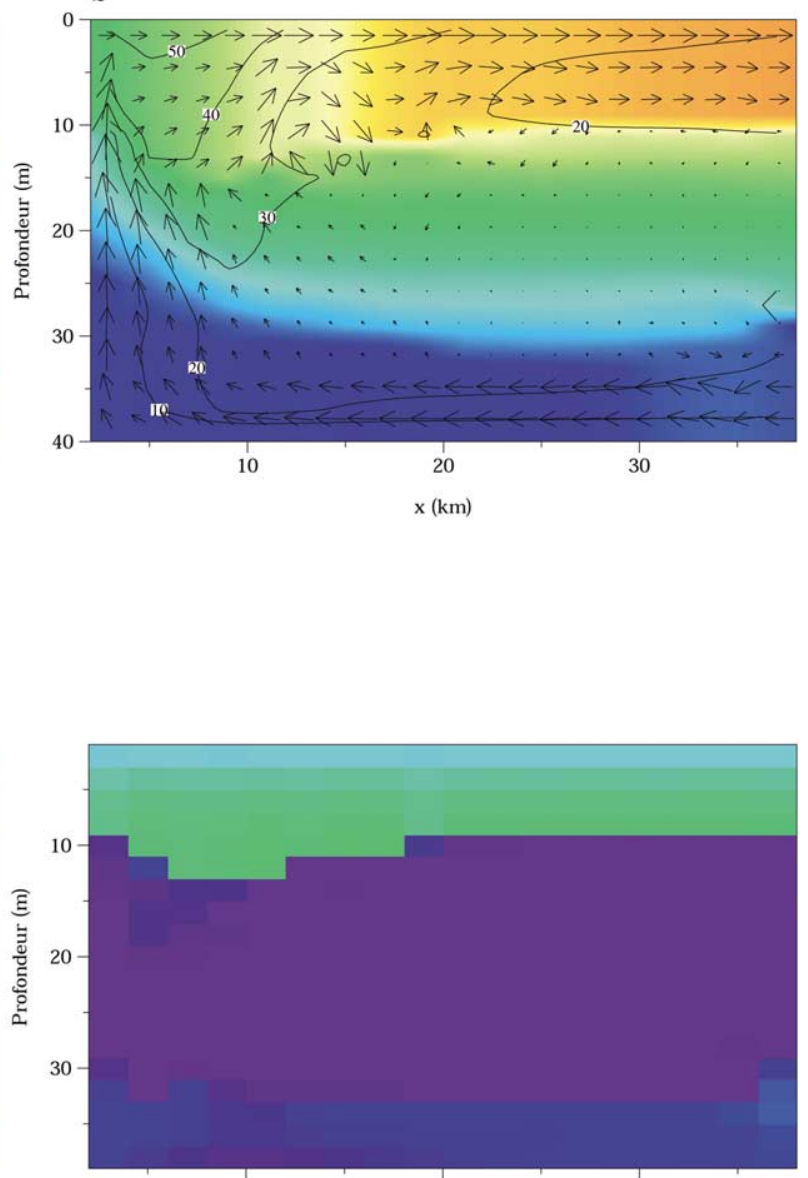

10

30

$\mathrm{x}(\mathrm{km})$

Figure 8. Expérience d'optimisation du champ de viscosité turbulente dans le cas d'un upwelling côtier. a. Observations simulées au moyen du modèle $k+L$. b. Solution du modèle après optimisation et son jeu de coefficients optimaux de viscosité turbulente.

relle pour répondre à cette problématique consiste à tenter d'optimiser directement le nombre restreint de constantes numériques intervenant dans le modèle de fermeture de la turbulence lui-même, en assimilant des observations des champs moyens hydrologiques ou dynamiques. L'utilisation de cette démarche pour accéder à une information de meilleure qualité sur les champs des moments et des flux turbulents parait, elle aussi, une voie de recherche intéressante à explorer Leredde et al, 2000).

D'une façon générale, l'optimisation des paramètres d'échange caractérisant les mouvements de petite échelle (turbulence) à partir d'observations relatives à des échelles d'ordre plus élevé, est un débat important dans la 
prise en compte des processus dans les modèles de circulation côtière, notamment au voisinage des couches limites de surface (effets de l'agitation due aux vagues) et de fond (paramètrisation des phénomènes de remise en suspension). Par ailleurs, l'identification de coefficients de diffusivité appropriés peut également être envisagée pour des espèces chimiques ou biologiques.

\section{RÉFÉRENCES}

Arakawa, A., Lamb, V.R., 1977. Computational design of the basic dynamical processes of the UCLA general circulation model, Method. Comput. Phys. 17, 173-265.

Begis, D., Crepon, M., 1975. On the generation of currents by wind: an identification method to determine Oceanic parameters. Lecture Notes in Physics No. 58.

Bennett, A., 1992. Inverse Method in Physical Oceanography. Cambridge University Press, Cambridge.

Bosseur, F., 1998. Détermination de conditions aux limites en mer ouverte avec une méthode de contrôle optimal. Thèse de doctorat, université de Corse.

Brauner, C.M., Penel, P., 1974. Identification of parameters in a nonlinear self consistent system including a Burgers' equation. J. Math. Anal. Appl. 45, 654-681.

Devenon, J.-L., 1986. Mesure de courants par radar VHF pour valider et optimiser les modèles numériques de circulation littorale en mer à marée. Thèse, université Paris-VI.

Devenon, J.L., 1990. Optimal control theory applied to an objective analysis of a tidal current mapping by HF Radar. J. Atmos. Ocean. Technol. 7, 269-284.

Gilbert, J.C., Lemarechal, C., 1989. Some numerical experiment with variable storage quasi-Newton algorithm. Math. Program. 45, 407-435.

Leendertse, J.J., Alexander, C. Jr, Liu, S.K., 1977. A Three Dimensional Model for Estuaries and Coastal Seas: Principles of Computation. The RAND Corporation, California.

Le Dimet, F.X., Talagrand, O., 1986. Variational algorithms for analysis and assimilation of meteorological observations: Theoretical aspects. Tellus 38A, 97-110.
Lellouche, J.M., 1995. Méthodes numériques d'assimilation de données appliquée à la modélisation tridimensionnelle des écoulements en milieu peu profond. Thèse de doctorat, université Aix-MarseilleII.

Lellouche, J.M., Devenon, J.L., Dekeyser, I., 1994. Boundary control on Burgers' equation: a numerical approach. Comput. Math Appl. $28,33-44$.

Lellouche, J.M., Devenon, J.L., Dekeyser, I., 1998. Data assimilation by optimal control in a 3D coastal oceanic model: The problem of discretisation. J. Atmos. Ocean. Technol. 15, 470-481.

Leredde, Y., Lellouche, J.M., Devenon, J.-L., Dekeyser, I., 1998. On initial, boundary conditions and viscosity coefficient control for Burgers' equation. Int. J. Num. Method Fluids 28, 113-128.

Leredde, Y., 1999. Méthode d'assimilation de données par contrôle optimal appliquée à l'estimation de paramètres de diffusion dans un modèle 3D de circulation océanique. Thèse de doctorat, université Aix-Marseille-II.

Leredde, Y., Devenon, J.L., Dekeyser, I., 1999. Turbulent viscosity optimized by data assimilation. An. Geophys. 17, 1463-1477.

Leredde, Y., Devenon, J.-L., Dekeyser, I., 2000. Peut-on optimiser les constantes d'un modèle de turbulence marine par assimilation d'observations ? C. R. Acad. Sci. Earth Planetary Sci. 331, 405-412.

Lions, J.-L., 1968. Contrôle optimal des systèmes gouvernés par des opérateurs aux dérivées partielles. Dunod, Paris.

Lewis, J.C., Derbe, J.M., 1985. The use of adjoint equations to solve a variational adjustment problem with advective constraints. Tellus 37A, 309-322.

Sasaki, Y.K., 1970. Some basic formalism in numerical variational analysis. Mon. Weather Rev. 98, 875-883.

Sirkes, Z., Tziperman, E., 1997. Finite difference of adjoint or adjoint of finite difference? Mon. Weather Rev. 125, 3373-3378.

Talagrand, O., 1991. The use of adjoint equations in numerical modeling of the atmospheric circulation, Proceedings in Applied Mathematics (SIAM) on automatic differentiation of algorithms: Theory, implementation and Application, pp. 169-180.

Talagrand, O., Courtier, P., 1987. Variational assimilation of meteorological observations with the adjoint vorticity equation, part I: theory. Quat. J. R. Meteorol. Soc. 113, 1311-1328. 